\title{
COMMENTARY
}

\section{The role of inflammation in ICU-acquired weakness}

\author{
Chris Winkelman* \\ See related research by Weber-Carstens et al., http://ccforum.com/content/14/3/R119
}

\begin{abstract}
A pilot observational study by Weber-Carstens and colleagues contributes to a mechanistic explanation of the puzzling and complex phenomena of ICU-acquired weakness (ICU-AW). The authors suggest systemic, inflammatory-mediated pathology is the most significant risk factor for ICU-AW. While this finding is somewhat equivocal, it provides important direction for future investigations and illustrates the challenges of interpreting significance in small observational studies.
\end{abstract}

The pilot observational study by Weber-Carstens and colleagues [1] provides important contributions to a mechanistic explanation of the puzzling and complex phenomena of ICU-acquired weakness (ICU-AW). Earlier findings from this research group suggested that ICUAW is primarily a myopathy [2] and confirmed that initial pathology manifests, on average, 7 days after ICU admission among the most severely ill [2-4]. In the current subanalysis with 40 of the original 52 subjects, multiple factors were examined for association with myopathy: molecular (IL-6, C-reactive protein (CRP), and insulin growth factor binding protein (IGFBP)-1); serum osmolarity; medication use (norepinepherine, dobutamine, hydrocortisone, aminoglycosides, analgesics, sedatives and neuromuscular blocking agents); and multisystem factors (simplified acute physiology (SAPS-2) and sequential organ failure assessment (SOFA) scores).

The authors suggest systemic, inflammatory-mediated pathology is the most significant risk factor for ICU-AW. The results on IL-6 show that its effects are actually quite modest. While IL-6 had a significant contribution to the statistical model, the hazard ratio of 1.006 indicated a higher IL-6 ( $>230$ picograms $/ \mathrm{ml}$ ) is little better than chance in predicting inexcitable muscle membranes (see

*Correspondence: Chris.Winkelman@case.edu

Frances Payne Bolton School of Nursing, Case Western Reserve University, 10900 Euclid Ave, Cleveland, OH 44106, USA
Figure 7 in [1]). CRP, the second inflammatory biomarker, was not associated with abnormal muscle excitability $(P=0.075)$. However, the sample size for this analysis was small. It may simply be that there are insufficient numbers of results to derive a meaningful Cox regression equation - allowing a reasonable 10 samples per factor/ covariate, a sample size of 160 would provide more value to the statistical model (16 covariates; see Table 2 in [1]).

The authors also report a hazard ratio for norepinepherine similar to that for IL-6. Along with the relative differences in the presence of septic shock and organ dysfunction in Table 1 in [1] among participants with/ without inexcitable muscle membrane, this finding lends support to oxidative stress or the interaction of oxidative stress and pro-inflammatory biomarkers as risk factors for myopathy in ICU patients [5]. Findings from this study illustrate the challenges of translating basic science to clinical settings. Multiple measures and more complex clinical data, such as a heterogeneous sample as in this report, make it difficult to derive important conclusions from small samples.

Building a framework to identify ICU-AW early and to evaluate efficacy of treatments is essential. Between 25 and $50 \%$ of patients who receive mechanical ventilation for 7 or more days experience neuromuscular abnormalities and these abnormalities can result in weakness and impaired function years after discharge from the ICU [6]. In the United States, from 1997 to 2006, the number of ICU patients who received mechanical ventilation and were subsequently discharged to home has decreased while transfers to long-term acute care increased significantly without concomitant changes in survival [7]. For older adults discharged with new or additional dependency in daily activities after hospitalization, less than $31 \%$ return to prehospital function [8]. Determining interventions that alter muscle pathology and associated dysfunction among patients who experience prolonged mechanical ventilation, whether from a mechanistic or a holistic perspective, has the potential to reduce the duration of mechanical ventilation and length of hospital stay [9].

The role of IL- 6 and other cytokines in muscle dysfunction is not yet clear. In healthy adults, very high levels can be myogenic after intense exercise [10]. Yet 
IL-6 is also associated with proteolysis and myosin loss [11]. Among patients with chronic inflammatory conditions/diseases, serum IL-6 is related to muscle wasting and dysfunction [12]. In ICU patients, IL-6 can be unconnected to illness severity yet predictive of mortality $[13,14]$. The sources of IL-6 - muscle versus leukocyte - may also be important to muscle pathology.

Developing understanding of basic pathology and establishing predictive biomarkers will provide the opportunity for new hypothesis testing. In this exploratory report of risk factors associated with abnormal responses to direct muscle stimulation, molecular to multisystem levels of covariates were examined [1]. Future studies will be more compelling when focused on single-level, inter-related pathways. Investigations related to molecular cascade interactions are providing insight into the genetic, signaling, bioenergetic, and metabolic processes that contribute to muscle health and disease. Understanding of molecular determinants of common diseases encountered in the critically ill can provide the rationale for selection of therapeutic targets [15]. If a serum IL-6 value $>230$ picograms $/ \mathrm{ml}$ is confirmed in future studies as an early indicator of muscle dysfunction, then the efficacy of prevention and treatment strategies may be measured rapidly and inexpensively by IL-6.

Observational data like this report provide important information with which to calculate effect size and determine promising biologic pathways for future investigations. Results also suggest that the timing of interventions to prevent ICU-AW may need to occur earlier than typically occurs in many settings as serum IL-6 and muscle stimulation responses were abnormal quite early in the majority of patients who went on to manifest ICU-AW.

\section{Abbreviations}

$C R P=C$-reactive protein; ICU-AW = ICU-acquired weakness; IGFBP = insulin growth factor binding protein; $I \mathrm{~L}=$ interleukin; SAPS = simplified acute physiology; SOFA = sequential organ failure assessment.

\section{Competing interests}

CW has received research and travel funding from Hill-Rom. 2007-2009.

\section{Acknowledgements}

Samantha Razavi, RN and Jeffrey Ruf, RN reviewed the literature and assembled articles for this commentary.
Published: 3 August 2010

\section{References}

1. Weber-Carstens S, Deja M, Koch S, Spranger J, Bubser F, Wernecke KD, Spies $C D$, Spuler S, Keh D: Risk factors in critical illness myopathy during the early course of critical illness: a prospective observational study. Crit Care 2010, 14:R119.

2. Weber-Carstens S, Koch S, Spuler S, Spies CD, Bubser F, Wernecke KD, Deja M: Nonexcitable muscle membrane predicts intensive care unit-acquired paresis in mechanically ventilated, sedated patients. Crit Care Med 2009, 37:2632-2637.

3. Friedrich O: Critical illness myopathy: What is happening? Curr Opin Clin Nutr Metab Care 2006, 9:403-409.

4. Fan E, Zanni JM, Dennison CR, Lepre SJ, Needham DM: Critical IIIness neuromyopathy and muscle weakness in patients in the intensive care unit. AACN Advanced Crit Care 2009, 20:243-253.

5. Reid MB, Andrade FH, Balke CW, Esser KA: Redox mechanisms of muscle dysfunction in inflammatory disease. Phys Med Rehabil Clin N Am 2005, 16:925-949.

6. Griffiths RD, Hall JB: Intensive care unit-acquired weakness. Crit Care Med 2010, 38:779-787.

7. Kahn JM, Benson NM, Appleby D, Carson SS, Iwashyna TJ: Long-term acute care hospital utilization after critical illness. JAMA 2010, 303:2253-2259.

8. Boyd CM, Landfeld CS, Counsell SR, Palmer RM, Fortinsky RH, Dresevic D, Curant C, Covinsky KE: Recovery of activities of daily living in older adults after hospitalization for acute medical illness. J Am Geriatric Soc 2008, 56:2171-2179.

9. Vincent J-L, Norrenberg M: Intensive care unit-acquired weakness: Framing the topic. Crit Care Med 2009, 37:S296-287.

10. Nielsen S, Pedersen BK: Skeletal muscle as an immunogenic organ. Curr Opin Pharmacol 2008, 8:346-351.

11. Truong AD, Fan E, Brower RG, Needham DM: Bench-to-bedside review: Mobilizing patients in the intensive care unit - from pathophysiology to clinical trials. Crit Care 2009, 13:216-224.

12. Morley JE, Thomas DR, Wilson M-MG: Cachexia: pathophysiology and clinical relevance. Am J Clin Nutr 2006, 83:735-743.

13. Levitt JE, Gould MK, Ware LB, Matthay MA: The pathogenetic and prognostic value of biologic markers in acute lung injury. I Intensive Care Med 2009, 24:151-167.

14. Kellum JA, Kong L, Fink MP, Weissfeld LA, Yealy DM, Pinsky MR, Fine J, Kirchevsky A, Delude RL, Angus DC: Understanding the inflammatory cytokine response in pneumonia and sepsis. Arch Intern Med 2007, 167:1655-1663.

15. Grimaldi D, Claessens Y-E, Mira J-P, Chiche J-D: Beyond the clinical phenotype: the biologic integratome. Crit Care Med 2008, 37:\$38-49.

doi:10.1186/cc9187

Cite this article as: Winkelman C: The role of inflammation in ICU-acquired weakness. Critical Care 2010, 14:186. 